\title{
Der Fiskalvertrag - ein Ausdruck deutscher Hegemonie in der Europäischen Union?
}

\author{
David Schäfer*
}

\begin{abstract}
„Deutschland entscheidet nicht für das gesamte Europa“1 - die Entrüstung von François Hollande wenige Tage vor seiner Wahl zum französischen Präsidenten über Deutschlands Rolle in der Schuldenkrise drückt pointiert ein Unwohlsein aus, das vielfach in der politischen Debatte und öffentlichen Meinung in anderen EU-Staaten artikuliert wird und zu der Frage führt: Ist Deutschland ein Hegemon in der Europäischen Union während der Schuldenkrise?
\end{abstract}

Ausgangspunkt der Erörterung ist das Verständnis von Hegemonie nach Heinrich Triepel, auf das auch Vertreter der neorealistischen Schule der Gegenwart, wie etwa Werner Link und Christoph Schönberger, ${ }^{2}$ aufbauen. Triepel definiert Hegemonie als „bestimmenden

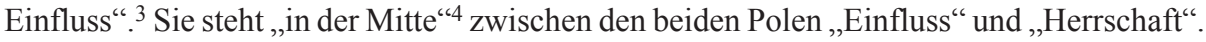
Der „bestimmende Einfluss“ des Hegemons geht also einerseits über die bloße Einflussnahme hinaus; er reicht jedoch nicht an die Herrschaft über den vom Hegemon geführten Staat heran. ${ }^{5}$ Hegemonie gefasst als ,bestimmender Einfluss“ unterscheidet sich von Herrschaft, indem sie nicht auf Zwang oder deren Androhung beruht, sondern das Einverständnis des dem Hegemon folgenden Staates voraussetzt. Von bloßem Einfluss unterscheidet sich die

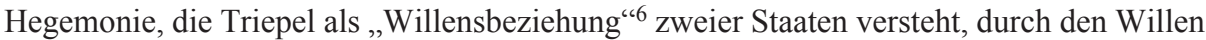
des Hegemons, eben jene hegemoniale Rolle wahrzunehmen. Zusammengefasst konstituiert sich Hegemonie daher aus drei Dimensionen: erstens bedarf sie materieller Überlegenheit eines Staates, um die Kosten einer hegemonialen Stellung zu übernehmen. Zweitens bedarf sie des Willens zur Wahrnehmung einer hegemonialen Rolle. ${ }^{7}$ Drittens basiert die hegemoniale Rolle auf der Akzeptanz des führenden Staats durch die ihm folgenden Staaten. ${ }^{8}$

Die Wahrnehmung einer hegemonialen Stellung ist in der Europäischen Union besonderen Bedingungen unterworfen. Hervorzuheben ist die zentrale Bedeutung der Mitgliedstaaten, die insbesondere durch den Europäischen Rat und den Rat maßgeblich in die Politikgestaltung eingebunden sind. Während von einer weitgehenden Verselbstständigung der supranationalen Ebene nur bedingt gesprochen werden kann, agieren die Mitgliedstaaten weiterhin als ,Herren der Verträge'. Diese Abwesenheit einer autonom regelsetzenden Bundesgewalt begünstigt die Herausbildung einer hegemonialen Struktur unter den Mitgliedstaaten. Wenngleich alle formal gleichgestellt sind, verhindert dies jedoch nicht, dass der Einfluss der Mitgliedstaaten faktisch variiert. Die für Verhandlungen in der Europäischen Union typische

* David Schäfer, Student, College of Europe, Brügge.

1 Le Figaro: Hollande: “L’Allemagne ne décide pas”, Lefigaro.fr, 26.4.2012.

2 Christoph Schönberger: Hegemon wider Willen: Zur Stellung Deutschlands in der Europäischen Union, in: Merkur 1/2012, S. 1-8; Werner Link: Integratives Gleichgewicht und gemeinsame Führung. Das europäische System und Deutschland, in: Merkur 11/2012, S. 1025-1034, hier S. 1025.

3 Heinrich Triepel: Die Hegemonie. Ein Buch von führenden Staaten, Aalen 1938, S. 40.

4 Ebenda, S. 40.

5 Ebenda, S. 40, 131, 141

6 Ebenda, S. 18.

7 Ebenda, S. 18-19.

8 Ebenda, S. 174-176. 
Konsenskultur verdeutlicht, dass hegemoniales Handeln der Akzeptanz durch die anderen Mitgliedstaaten bedarf. Zwar strebt der Hegemon nach Durchsetzung seiner eigenen Anliegen, muss dennoch stets die Interessen der anderen Mitgliedstaaten bedienen. ${ }^{9}$ In der vorliegenden Studie wird die Akzeptanz deutscher Hegemonie anhand von Frankreich, Spanien und Irland analysiert, da sie repräsentativ die Unterschiede in Landesgröße, Bevölkerung und wirtschaftlicher Leistungsfähigkeit ${ }^{10}$ unter den Mitgliedstaaten der Europäischen Union abbilden. Der Untersuchungszeitraum reicht von den Anfängen der Schuldenkrise im Oktober 2009 mit dem Eingeständnis eines massiven Budgetdefizits seitens der griechischen Regierung ${ }^{11}$ und endet am 2. März 2012 mit der Unterzeichnung des „Vertrags über Stabilität, Koordinierung und Steuerung in der Wirtschafts- und Währungsunion“"(Fiskalvertrag). Dieses Vertragswerk wurde gewählt, da es sowohl als hochgradig relevanter Beitrag zur Krisenbewältigung angesehen wird ${ }^{12}$ als auch aufgrund des intergouvernementalen Verhandlungsprozesses den Einfluss einzelner Mitgliedstaaten abbildet.

\section{Die Wirtschaftsleistung im Vergleich: materielle Machtfaktoren}

Die Schuldenkrise offenbarte über viele Jahre andauernde Fehlentwicklungen in der Eurozone, die maßgeblich die wirtschaftliche Leistungsfähigkeit der Mitgliedstaaten in der Europäischen Union beeinflussten. Während sich die deutsche Wirtschaft als wettbewerbsfähig erwies, sahen sich insbesondere die sogenannten GIIPS (Griechenland, Irland, Italien, Portugal und Spanien) enormen Problemen ausgesetzt und kämpften mit massiven Schwierigkeiten der Staatsfinanzierung. Die problematische Entwicklung einiger Mitgliedstaaten verdüsterte das Vertrauen der Marktteilnehmer. Während Deutschland sich zeitweise sogar mit Negativzinsen finanzieren konnte, war es in erster Linie das Eingreifen der Europäischen Zentralbank, das Spanien und Italien vor dem Staatsbankrott rettete. Andere Staaten - wie Griechenland, Irland, Portugal sowie zur Refinanzierung der Banken auch Spanien - griffen auf Gelder aus dem Europäischen Stabilitätsmechanismus (ESM) zurück. Die systemrelevante Position Deutschlands wurde im Zuge der Krisenpolitik offensichtlich (siehe hierzu auch Tabelle 1). ${ }^{13}$ Zwar ist die Bundesrepublik nicht der einzige Staat der Europäischen Union mit einer soliden Haushaltssituation, in ihrem Falle geht diese jedoch mit der schieren Größe des Staates einher. Kein anderer Mitgliedstaat verfügt über eine vergleichbare, auf der Wirtschaftsleistung beruhenden, Machtposition. ${ }^{14}$ Ein prägnantes Beispiel ist der neugeschaffene ESM, der mit 700 Milliarden Euro Stammkapital ${ }^{15}$ ein Herzstück der neuen Architektur der Eurozone darstellt. Mit einem Anteil von 27,15 Prozent ${ }^{16}$ des Stammkapitals, der von Deutschlands ,AAA'-Rating abgedeckt wird, übernimmt Deutschland die Rolle des wichtigsten Geldgebers. Eine solide Kreditbewertung der Bundesrepublik ist unabdingbar für die effektive Nutzung des Instruments, dessen Finanzierungskosten sich andernfalls er-

9 Schönberger: Hegemon wider Willen, 2012, S. 2-3.

10 Jonas Tallberg: Bargaining Power in the European Council, in: Journal of Common Market Studies 3/2008, S. 685-708, hier S. 689.

11 Tobias Kunstein/Wolfgang Wessels: Die Europäische Union in der Währungskrise: Eckdaten und Schlüsselentscheidungen, in: integration 4/2011, S. 308-322, hier S. 309.

12 Ebenda, S. 320-322.

13 Link: Integratives Gleichgewicht und gemeinsame Führung, 2012, S. 1025.

14 Schönberger: Hegemon wider Willen, 2012, S. 1.

15 Bundesfinanzministerium: Fragen und Antworten zum Europäischen Stabilisierungsmechanismus, 26.9.2012, abrufbar unter: http://www.bundesfinanzministerium.de/Content/DE/FAQ/2012-08-16-esm-faq.html (letzter Zugriff: 10.3.2013).

16 Ebenda. 
höhen. Die ökonomische Unverzichtbarkeit transformierte sich in politisches Kapital. Deutschland wurde in der Schuldenkrise faktischer Veto-Spieler.

Tabelle 1: Indikatoren wirtschaftlicher Leistungsfähigkeit während der Verhandlungen zum Fiskalvertrag (Jahr 2012)

\begin{tabular}{|l|l|l|l|l|}
\hline & \multicolumn{1}{|c|}{ Deutschland } & \multicolumn{1}{|c|}{ Frankreich } & \multicolumn{1}{c|}{ Spanien } & \multicolumn{1}{|c|}{ Irland } \\
\hline BIP (Eurozone) ${ }^{17}$ & $27,8 \%$ & $21,4 \%$ & $11,1 \%$ & $1,7 \%$ \\
\hline Kreditbewertung $^{18}$ & & & & \\
\hline Fitch & AAA & AAA & AA- & BBB+ \\
\hline Moody's & AAA & AAA & Baa3 & Ba1 \\
\hline Standard \& Poor's & AAA & AA + & BBB + & BBB + \\
\hline $\begin{array}{l}\text { Zinsrate Staatsanleihen } \\
\text { (1. Quartal) }^{19}\end{array}$ & $1,83 \%$ & $3,05 \%$ & $5,23 \%$ & $\begin{array}{l}7,7 \% \\
(4 . \text { Quartal } \\
2010)\end{array}$ \\
\hline $\begin{array}{l}\text { Ranking Wettbewerbs- } \\
\text { fähigkeit }\end{array}$ & 6 & 21 & 36 & 27 \\
\hline
\end{tabular}

Quelle: Eigene Darstellung auf Basis der angegebenen Quellen.

Kann daraus eine hegemoniale Rolle abgeleitet werden? Richtet man diese Bewertung an dem oben eingeführten Kriterium des „bestimmenden Einflusses“ aus, so ist diese Frage zu bejahen. Deutschland „gibt den Takt vor“, 21 ein effektives Krisenmanagement ist von der Zustimmung der deutschen Regierung abhängig. Die wirtschaftliche Macht Deutschlands kann jedoch nur schwerlich von den Interdependenzen innerhalb der Europäischen Union isoliert betrachtet werden. ${ }^{22}$ Von besonderer Bedeutung für Deutschland sind die Außenhandelsbeziehungen. 57,1 Prozent der deutschen Exporte wurden 2012 innerhalb der Europäischen Union abgesetzt. Der Anteil der EU-Importe belief sich auf 63,5 Prozent. ${ }^{23}$ Aus diesen gegenseitigen Abhängigkeiten ergeben sich Einschränkungen der aus der Wirtschaftskraft erwachsenden politischen Macht. Zudem erhöht die Interdependenz das Interesse, kooperativ Problemlösungen zu finden. Eine Schlüsselrolle kommt Frankreich zu, das über ein Fünftel des Bruttoinlandsprodukts der Eurozone erwirtschaftet und Deutschlands wichtigster Handelspartner innerhalb der Europäischen Union ist. Es befindet sich bezüglich Landesgröße, Bevölkerungszahl und Wirtschaftskraft in ähnlichen Dimensionen wie

17 Bruttonationaleinkommen der Mitgliedstaaten als Anteil am BIP der Eurozone. Daten von Eurostat-Onlinedatenbank, abrufbar unter: http://epp.eurostat.ec.europa.eu/portal/page/portal/eurostat/home/ (letzter Zugriff: 13.5.2013).

18 Fitch: Fitch Places Belgium, Spain, Slovenia, Italy, Ireland and Cyprus on Rating Watch Negative, 16.12.2011; Fitch: France, 16.12.2011; Fitch: Germany, 20.9.2011; Moody’s: France. Government of, 15.12.2011; Moody's: Germany. Government of, 15.12.2011; Moody's: Ireland. Government of, 15.12.2011; Moody's: Spain. Government of, 15.12.2011; S\&P: France. Republic of, 13.1.2012, S\&P: Germany. Federal Republic of, 13.1.2012; S\&P: Ireland. Republic of, 13.1.2012; S\&P: Spain. Kingdom of, 13.1.2012.

19 Eurostat-Onlinedatenbank.

20 Klaus Schwab (Hrsg.): World Economic Forum. The Global Competitiveness Report 2012-2013, Geneva 2012, S. 13.

21 Peter Ludlow: Prospects for the March European Council, EuroComment Briefing Note, Brüssel 2012, S. 5.

22 Link: Integratives Gleichgewicht und gemeinsame Führung, 2012, S. 1028.

23 Eurostat-Onlinedatenbank. 
Deutschland. Das traditionelle französische Streben nach einer Führungsrolle wird zumeist im Rahmen des deutsch-französischen Tandems realisiert. Die Stellung Deutschlands entspricht also vielmehr einer regionalen Hegemonialmacht mit Bedarf nach Kooperationspartnern.

\section{Identität und Fremdwahrnehmung: immaterielle Machtfaktoren}

\section{Deutschland - kein Wille zur Hegemonie?}

Ist Deutschland in der Schuldenkrise gewillt, die ökonomischen Ressourcen in eine hegemoniale Rolle zu transformieren? Der Ruf nach einer aktiveren Rolle Deutschlands ist zumindest in einigen Mitgliedstaaten, so durch den polnischen Außenminister Radosław Sikorski, oder in internationalen Foren wie den G-20 deutlich artikuliert worden. ${ }^{24}$ Als problematisch erweisen sich nach Schönberger die „mentalen und institutionellen Barrieren“, 25 die einer hegemonialen Rolle Deutschlands entgegenstehen. Vertreter konstruktivistischer Ansätze argumentieren, eine deutsche Hegemonie sei nicht mit der durch die traumatischen Erlebnisse der Nazi-Herrschaft geprägten deutschen Staatsraison vereinbar. ${ }^{26}$ Pointiert drückt dies der deutsche Finanzminister Wolfgang Schäuble aus: „Als Europäer, der in der Nachkriegszeit aufgewachsen ist, bin ich jedoch davon überzeugt, dass das Verlangen nach einer ausgeprägten Führungsrolle von Deutschland in Europa nicht besonders klug ist “. ${ }^{27}$ Während deutsche Politik im Ausland zumeist als ,bestimmender Einfluss' angesehen wird, ist diese Position im deutschen Bewusstsein nur mangelhaft verankert. ${ }^{28}$ Der Handlungsspielraum der Bundesregierung auf europäischer Ebene wird zudem vermehrt von nationalen Institutionen eingeschränkt. Jede mühsam ausgehandelte Kompromisslösung zwischen den Regierungen muss dem kritischen Urteil des Bundesverfassungsgerichts standhalten. Unsichere Mehrheiten im Bundestag führen zu einem engen Mandat für Regierungsvertreter bei zwischenstaatlichen Verhandlungen. Dies zwingt die Bundesregierung zu einem Diskurs, der die Wahrung nationaler Interessen gegenüber Begehrlichkeiten anderer Mitgliedstaaten betont. Dies ist schwerlich mit den Anforderungen an einen,wohlwollenden Hegemon` vereinbar. Ein bekanntes Muster zur Überwindung dieses Dilemmas bildete im Integrationsverlauf das deutsch-französische Tandem. Eine kooperativ mit dem einstigen Erzfeind Frankreich wahrgenommene Hegemonie ${ }^{29}$ erwies sich als probates Mittel, den Handlungsspielraum Deutschlands auf europäischer Ebene zu erweitern, ohne Widerstände im In- wie Ausland gegenüber einer argwöhnisch betrachteten unilateralen Führungsrolle zu wecken. Auch in der Schuldenkrise ist die Zusammenarbeit mit Frankreich ein Kernanliegen der deutschen Bundesregierung. In einer bemerkenswerten Rede an der Pariser Universität La Sorbonne charakterisierte Schäuble die deutsch-französische Zusammenar-

24 Radosław Sikorski: „I fear German power less than German inaction“, Berlin-Rede bei der DGAP, 2011, abrufbar unter: https://dgap.org/en/node/20055 (letzter Zugriff: 28.1.2013); Ignazio Angeloni: Reflections after the G20 meeting in Mexico, Bruegel, 2012, abrufbar unter: http://www.bruegel.org/blog/detail/article/690-refl ections-after-the-g20-meeting-in-mexico/ (letzter Zugriff: 28.1.2013).

25 Schönberger: Hegemon wider Willen, 2012, S. 4.

26 Martin Marcussen/Thomas Risse/Daniela Engelmann-Martin/Hans Joachim Knopf/Klaus Roscher: Constructing Europe? The Evolution of French, British, and German Nation-State Identities, in: Journal of European Public Policy 4/1999, S. 614-633, hier S. 622-625.

27 Stuttgarter Zeitung: Der Bundesfinanzminister warnt davor, vom eingeschlagenen Weg zur Euro-Rettung abzuweichen, Interview mit Wolfgang Schäuble, 27.1.2012.

28 Schönberger: Hegemon wider Willen, 2012, S. 4.

29 Thomas Pedersen: Cooperative Hegemony: Power, Ideas and Institutions in Regional Integration, in: Review of International Studies 4/2002, S. 677-696. 
beit unter Rückgriff auf die Theorie hegemonialer Stabilität. Gemäß diesem Ansatz wirkt ein Hegemon als Stabilisator internationaler Kooperation. Ein hegemoniales Verhältnis ist demnach auch für die folgenden Staaten gewinnbringend, da der Hegemon ein öffentliches Gut bereitstellt. ${ }^{30}$ Schäuble porträtierte Deutschland und Frankreich in seiner Rede als gemeinsamen Hegemon in der Europäischen Union während der Schuldenkrise. ${ }^{31}$ Eine alleinige hegemoniale Rolle Deutschlands steht auch während der Schuldenkrise unter der Einschränkung, nur in einem multilateralen Kontext stattfinden zu können. Unilaterale Handlungen hingegen widersprechen der Staatsraison und stellen folglich keine Handlungsoption dar. $^{32}$

\section{Frankreich - ein unwilliger Juniorpartner?}

Wie reagiert die französische Regierung auf die in der Schuldenkrise deutlich zu Tage getretene materielle Stärke Deutschlands und die daraus resultierenden veränderten Kräfteverhältnisse im deutsch-französischen Tandem? Das Verhältnis zu Deutschland ist stetiger Fixpunkt der französischen Staatsraison. Entsprechend rührt eine Re-Konfiguration der Machtverteilung zwischen beiden Staaten am Selbstverständnis Frankreichs. ${ }^{33}$ Eine Führungsrolle, wie sie französische Regierungen über den gesamten Integrationsprozess beanspruchten, bedarf einer materiellen Basis. Daher stellt die gegenüber Deutschland schwächere Wirtschaftsentwicklung die Grundzüge der französischen Identität und des Wirkens in der Europäischen Union infrage. Wenngleich Frankreich wirtschaftlich den Anschluss an Deutschland zunächst verlor, gibt es bisher allerdings wenige Anzeichen, dass es sich mit einer daraus eventuell abzuleitenden Rolle als Juniorpartner zufrieden gäbe. Wie zuvor schon im Falle Deutschlands ist ein Missverhältnis zwischen materiellen Rahmenbedingungen Frankreichs und dem Selbstverständnis der Entscheidungsträger in Paris zu konstatieren. Während die traditionellen ,Insignien` französischer Macht wie der Ständige Sitz im Sicherheitsrat der Vereinten Nationen oder der Status als Atommacht im Zuge der EU-Krisenbewältigung von geringer Bedeutung sind, kann Frankreich aufgrund wirtschaftlicher Schwäche hierzu nur bedingt beitragen. ${ }^{34}$ Die Fixierung auf die relative Machtposition zu Deutschland steht der Akzeptanz einer deutschen hegemonialen Rolle entgegen, macht diese fast unmöglich. Zwar bezog sich der damalige französische Präsident Nicolas Sarkozy mit Reformvorhaben in den Bereichen Arbeitsmarkt, Soziales und Steuerpolitik explizit auf Deutschland und die unter Bundeskanzler Gerhard Schröder durchgeführten Reformen. ${ }^{35}$ Wie jedoch die teils harschen Reaktionen in der französischen Öffentlichkeit auf diese Orientierung an Deutschland zeigten, kann hieraus nur schwerlich die Duldung einer Deutschland nachgeordneten Rolle abgeleitet werden. Die Bundesrepublik wird zwar als ökonomisch

30 Siehe Robert O. Keohane: After Hegemony, Princeton 1984.

31 Wolfgang Schäuble: Rede des Bundesministers der Finanzen Dr. Wolfgang Schäuble an der Université ParisSorbonne, 2.11.2010, abrufbar unter http://www.bundesfinanzministerium.de/Content/DE/Reden/2010/2010-1 1-02-sorbonne.html (letzter Zugriff: 12.1.2013).

32 William Paterson: The Reluctant Hegemon? Germany moves centre stage in the European Union, in: Journal of Common Market Studies, Annual Review 2011, S. 57-75, hier S. 65-68.

33 Marcussen/Risse/Engelmann/Knopf/Roscher: Constructing Europe?, 1999, S. 619.

34 Schönberger: Hegemon wider Willen, 2012, S. 3.

35 Nicolas Sarkozy: Rede in Lyon, 19.1.2012, abrufbar unter: http://www.archives.elysee.fr/president/les-actualit es/discours/2012/discours-a-l-occasion-des-voeux-aux-forces.12835.html (letzter Zugriff: 21.6.2012). 
überlegen und wettbewerbsfähiger anerkannt, als Preisgabe einer politischen Führungsrolle der ,grande nation' kann dies jedoch nicht interpretiert werden. ${ }^{36}$

\section{Spanien - mit Deutschland aus der Krise?}

Wie positioniert sich Spanien gegenüber einer deutschen Hegemonie? Sowohl der ehemalige Ministerpräsident José Luis Rodríguez Zapatero als auch sein Nachfolger Mariano Rajoy machten Deutschland während des Untersuchungszeitraums zum Dreh- und Angelpunkt spanischer Europapolitik. Hiermit einher ging Zapateros Drängen nach einer aktiveren deutschen Rolle. ${ }^{37}$ Sein Handeln basierte auf der Annahme, die Unterstützung Deutschlands zu benötigen, um spanische Interessen durchzusetzen. Ziel war es, durch entschlossenes Vorgehen ein Signal der Handlungsfähigkeit an die Märkte zu senden und die Finanzierungsschwierigkeiten zu überwinden. Die Mitgliedschaft in der Eurozone stand indes nie zur Disposition: Ein Verlassen der Eurozone wäre gleichbedeutend gewesen mit einem Rückfall in den ,atraso', die Rückständigkeit Spaniens wie zu Zeiten des Franco-Regimes. ${ }^{38}$ Die 2011 gewählte Regierung von Rajoy versprach daher alle nötigen Anstrengungen, um den Staat und die Europäische Union aus der misslichen Lage zu befreien: „Wir werden der loyalste und auch der ehrgeizigste Mitgliedstaat sein. [...] Lasst uns aufhören, ein Teil des Problems zu sein. Wir wollen wieder Teil der Lösung sein“. ${ }^{39}$ Deutschland kam hierbei - an eine lange Tradition anknüpfend ${ }^{40}$ - die Rolle als wirtschaftlicher Modellstaat zu. Wenngleich Spanien insbesondere nach der Wahl von Hollande zum französischen Präsidenten im Mai 2012 vermehrt Kritik an deutschen Positionen vernehmen ließ, stand die Regierung von Rajoy während der Verhandlungen zum Fiskalpakt noch in enger Partnerschaft zur deutschen Regierung. Diese Anziehungskraft Deutschlands und das Bemühen Spaniens, Reformen in dem von Deutschland geforderten Sinne umzusetzen, deuten auf eine grundsätzliche Akzeptanz deutscher Hegemonie im Untersuchungszeitraum.

\section{Irland - ein gern gesehener Musterschüler?}

Wie bewertet die irische Regierung einen Hegemon Deutschland? Der Stellenwert der Beziehungen zu Deutschland war für Irland nicht derart zentral wie im Falle Frankreichs oder Spaniens. Dennoch waren sich die Entscheidungsträger auch hier bewusst, dass Deutschland als faktische Vetomacht großen Einfluss auf den Verlauf der Krise in Irland ausübt. ${ }^{41}$ Nachdem der einstige ,keltische Tiger' massiv unter dem Zusammenbruch des heimischen Bankensektors litt, wurden in der Folge hohe Budgeteinsparungen mit schmerz-

36 Claire Demesmay/Yann-Sven Rittelmeyer: France's Partner on a Pedestal. A view driven by pragmatism and envy, in: Almut Möller/Roderick Parkes (Hrsg.): Germany as Viewed by Other EU Member States, European Policy Institutes Network: EPIN Paper 33/2012, S. 25-28, hier S. 26-27.

37 Frankfurter Allgemeine Zeitung: Zapatero: Spanien macht seine Hausaufgaben, Interview mit José Luis Zapatero, 2.2.2011, S. 5.

38 Pablo Jauregui: Spain: „Europe“ as a Symbol of Modernity, Democracy and Renewed International Prestige, in: Bo Stråth/Anna Triandafyllidou (Hrsg.): Representations of Europe and the nation in current and prospective member states. Media, elites and civil society, Brüssel 2003, S. 274-319, hier S. 292, 307.

39 Mariano Rajoy: Mensaje a la Nación de Mariano Rajoy, 21.11.2011, abrufbar unter: http://politica.elpais.com/ politica/2011/11/21/actualidad/1321833683 582220.html (letzter Zugriff: 26.1.2013).

40 Laia Mestres: The View from Spain. Between Euro-Enthusiasm and Euro-Austerity', in: Almut Möller/Roderick Parkes (Hrsg.): Germany as Viewed by Other EU Member States, European Policy Institutes Network: EPIN Paper 33/2012, S. 16-19, hier S. 16.

41 Brigid Laffan: Ireland and Europe 2010. An Unwelcome Critical Juncture?, Dublin 2010, S. 18. 
haften sozialen Folgen vorgenommen. ${ }^{42}$ In Irland paarte sich die Einsicht in die wirtschaftlichen Probleme mit dem Bewusstsein, diese in erster Linie selbst lösen zu müssen. Der irische Premierminister Enda Kenny gab sich ,zu 100 Prozent überzeugt, dass es das Beste ist, uns selbst zu helfen, indem wir unser EU/IWF-Anpassungsprogramm pünktlich und in Gänze durchführen“. ${ }^{43}$ Einhergehen die Sparmaßnahmen jedoch mit der Wahrnehmung, substanziell Souveränität eingebüßt zu haben. Als kleiner Mitgliedstaat der Europäischen Union sieht man sich in einer besonderen Abhängigkeit von den großen Mitgliedstaaten. Zusammenfassend lässt sich das Verhältnis Irlands zu einer deutschen Hegemonie als rein funktional im Sinne einer effektiven Krisenbewältigung zusammenfassen, dessen primäres Ziel es ist, die mit der desolaten Haushaltslage einhergehenden Einschränkungen finanzieller Souveränität zu überwinden.

\section{Der Fiskalvertrag als Fallstudie: ein Vertrag aus deutscher Feder?}

Der Fiskalvertrag wurde in den Grundzügen auf der Sitzung des Europäischen Rates am 8./9. Dezember 2011 beschlossen, am 2. März 2012 unterzeichnet und trat am 1. Januar 2013 in Kraft. Aufgrund eines Vetos des Vereinigten Königreichs gegenüber einer EU-Vertragsänderung ist er als völkerrechtlicher Vertrag gefasst, dem sich neben dem Vereinigten Königreich auch Tschechien nicht anschloss.

Formal gliedert sich der Fiskalvertrag in drei Bestandteile: Kern ist der ,fiskalpolitische Pakt". Hierin verpflichten sich die Staaten auf einen ausgeglichenen Haushalt. ${ }^{44}$ Liegt der staatliche Schuldenstand über 60 Prozent des Bruttoinlandsprodukts, darf das neu als Kriterium eingeführte „strukturelle Defizit ${ }^{645}$ eines Mitgliedstaates 0,5 Prozent des Bruttoinlandsprodukts nicht überschreiten. Die Unterzeichnerstaaten verpflichten sich, diese ,goldene Regel` zu einem ausgeglichenen Haushalt in ihre nationalen Rechtssysteme ,,vorzugsweise mit Verfassungsrang ${ }^{“ 46}$ zu übertragen. Überwacht wird dies durch den Gerichtshof der Europäischen Union. Klageberechtigt sind die Mitgliedstaaten, die Kommission hingegen nicht. ${ }^{47}$ Wird das Defizit-Kriterium von einem Staat verletzt, treten Sanktionen auf Empfehlung der Kommission nach dem neuen Regelwerk automatisch ein, sofern der Rat sich nicht mit qualifizierter Mehrheit dagegen ausspricht (,umgekehrte' qualifizierte Mehrheit) ${ }^{48}$ Im Falle des Schuldenkriteriums ist die aktive Zustimmung des Rates mit qualifizierter Mehrheit notwendig. ${ }^{49}$ Die Ratifizierung des Fiskalvertrags ist Voraussetzung für Finanzhilfen durch den Europäischen Stabilisierungsmechanismus. ${ }^{50}$ Mit der zweiten Säule des Fiskalvertrags wird eine stärkere wirtschaftspolitische Koordinierung der Eurozone angestrebt, ohne über vage Zielbestimmungen hinauszugehen. ${ }^{51}$ Der dritte Teil des Vertrages

42 Corinne Deloy: The Irish Largely Approve the European budgetary pact, Fondation Robert Schuman, Paris 2012, S. 2.

43 Enda Kenny: Rede zur Sitzung des Europäischen Rats vom 26.10.2011, 2.11.2011, abrufbar unter: http://www .merrionstreet.ie/index.php/2011/11/statement-by-the-taoiseach-following-the-meeting-of-the-european-coun cil-in-brussels-26-october-2011-in-dail-eireann-2-november-2011-2/?cat=70 (letzter Zugriff: 30.6.2012).

44 Art. 4 Vertrag über die Stabilität, Koordinierung und Steuerung in der Wirtschafts- und Währungsunion in der Fassung vom 2.3.2012 (Fiskalvertrag).

45 Art. 3 Abs. 1 Lit. b Fiskalvertrag.

46 Art. 3 Abs. 2 Fiskalvertrag.

47 Art. 8 Fiskalvertrag.

48 Art. 7 Fiskalvertrag.

49 Art. 4 Fiskalvertrag.

50 Erwägungsgründe Fiskalvertrag.

51 Art. 9-11 Fiskalvertrag. 
institutionalisiert die Bestimmungen über die Steuerung der Eurozone, wie sie bereits in den Schlussfolgerungen des Europäischen Rates am 26. Oktober $2011^{52}$ dargelegt wurden. Mindestens zweimal jährlich finden Euro-Gipfel statt, an denen die Staats- oder Regierungschefs der 17 Staaten der Eurozone sowie die Präsidenten der Kommission und der Europäischen Zentralbank teilnehmen. Mindestens einmal jährlich sind die Mitgliedstaaten, die nicht die gemeinsame Währung eingeführt haben, teilnahmeberechtigt. Dieser stark intergouvernementale Aufbau der institutionellen Struktur findet in der mit den Euro-Finanzministern besetzten Euro-Gruppe ${ }^{53}$ seine Fortsetzung.

\section{Deutschland - keine Hilfszahlungen ohne, goldene Regel ' und Konditionalität}

Maßnahmen auf europäischer Ebene zur Unterstützung der Haushaltsdisziplin kam eine Schlüsselrolle in der deutschen Strategie zu, um dauerhafte Transferzahlungen an fiskalisch unsolide Mitgliedstaaten zu vermeiden. Während der Verhandlungen drängte die Bundesregierung vehement auf eine Änderung des Primärrechts, um ein „starkes Signal“ an die Europäische Zentralbank, die Finanzmärkte und nicht zuletzt auch an die einheimische Bevölkerung zu senden. ${ }^{54}$ Aller Bemühungen um einen Konsens im Kreise der 27 Mitgliedstaaten zum Trotz war man jedoch auch bereit, im Falle eines möglichen Vetos eines oder mehrerer EU-Staaten, im ,EU-17+'-Rahmen zu agieren. Dies würde auf ein ,Ausspielen“ der systemrelevanten deutschen Position in der Europäischen Union hinweisen. Vor dem Hintergrund der obigen Analyse, die insbesondere die zurückhaltende deutsche Politik herausarbeitet, ist dieses Resultat zunächst überraschend; die aktive Suche nach dem Schulterschluss mit Frankreich unterstreicht jedoch wieder das kooperative Selbstverständnis, wie es Teil der Selbstwahrnehmung Deutschlands ist. Kernziel war die Aufnahme einer, goldenen Regel' in die nationalen Verfassungsordnungen, um dauerhaft fiskalische Disziplin zu gewährleisten. ${ }^{55}$ Vorbild war die seit 2009 im Grundgesetz verankerte ,Schuldenbremse‘. Zudem setzte Deutschland sich im Rahmen der Verhandlungen für eine starke Rolle des Gerichtshofs der Europäischen Union und der Kommission ein. Ersterer sollte nicht nur für die rechtliche Implementierung der Schuldenbremse zuständig sein, sondern auch deren Anwendung bezüglich des Schulden- und Defizitkriteriums überwachen. Deutschland strebte darüber hinaus an, der Kommission die Kompetenz zu übertragen, bei Verstößen gegen die Haushaltsziele des Fiskalvertrags den Gerichtshof anrufen zu können. Die ,umgekehrte‘ qualifizierte Mehrheit sollte gemäß der deutschen Position sowohl bei Verstößen gegen das Defizit- als auch gegen das Schuldenkriterium Anwendung finden. ${ }^{56}$ Hieraus lässt sich ein für deutsche Regierungen nicht untypischer regelbasierter Ansatz ableiten. Den insbesondere von Spanien ausgehenden Initiativen zur Ahndung exzessiver Exportüberschüsse wurde hingegen kategorisch widersprochen. ${ }^{57}$ Bemerkenswert ist die Zustimmung Deutschlands zu einer weitgehend intergouvernementalen Organisation der Eurozone. ${ }^{58}$ Folgt man den An-

52 Europäischer Rat: Erklärung des Euro-Gipfels vom 26.10.2011, 8.11.2013, SN 3993/5/11.

53 Art. 12 Fiskalvertrag.

54 Peter Ludlow: The European Council of 8/9 December 2011, EuroComment Briefing Note, Brüssel 2012, S. 7 , 14-15, 39-40.

55 Agence Europe: Budget Talks Make Good Progress, 11.1.2012.

56 Michael Kaczmarek: Ergebnisse des deutschen EU-Gipfels, in: EurActiv, 31.1.2012.

57 Daniela Schwarzer: Germany and the Euro. A Reluctant Leader?, in: Renaud Dehousse/Elvire Fabry (Hrsg.): Where is Germany heading?, Paris 2010, S. 13-20, hier S. 18-19; Agence Europe: $2^{\text {nd }}$ Greek Bailout and Reinforcement of Stability Pact on Menu, 18.2.2012.

58 Desmond Dinan: Governance and Institutions. Implementing the Lisbon Treaty in the Shadow of the Euro Crisis, in: Journal of Common Market Studies, Annual Review 2011, S. 103-121, hier S. 107. 
nahmen konstruktivistischer Ansätze, wäre eine Präferenz zu weiterer Supranationalisierung zu erwarten, wie sie ein Bestandteil des normativen Bekenntnisses zur europäischen Einigung darstellt. Tatsächlich plädierte Deutschland für Formen der Beteiligung der Euro-Staaten, die den Euro nicht eingeführt haben, an den Euro-Gipfeln. ${ }^{59}$ Unverzichtbar war es aus deutscher Sicht, die Ratifikation des Fiskalvertrags und die damit verbundene Haushaltsdisziplin zu einer Bedingung für die Vergabe von ESM-Mitteln zu machen. ${ }^{60}$

\section{Frankreich - der Primat der , volonté politique}

War Frankreich gegenüber einem hegemonialen Streben Deutschlands tatsächlich so skeptisch, wie die obigen Ausführungen zur Staatsraison erwarten lassen? Viele Elemente der Agenda der französischen Regierung während des Untersuchungszeitraums lassen sich auf das Bemühen verdichten, die Handlungsfähigkeit der Politik gegenüber den als irrational agierend empfundenen Märkten wieder herzustellen. Dieses Anliegen findet eine Entsprechung in einer intergouvernementalen Vision der zukünftigen Steuerung der Eurozone. ${ }^{61}$ So wurde dem Gerichtshof eine Rolle mit reduzierten Zuständigkeiten zugedacht, um ein ,gouvernement des juges “62 mit einer als zu gestaltend wahrgenommenen Rolle der Luxemburger Institution zu verhindern. Vielmehr wurde darauf hingewiesen, dass die Verwaltung des Haushalts in nationaler Zuständigkeit verbleiben möge. ${ }^{63}$ Folglich lehnte die französische Regierung ein Klagerecht der Kommission vor dem Gerichtshof ab. Sie plädierte für das System der aktiven Zustimmung des Rates mit qualifizierter Mehrheit bei Verstößen gegen das Defizit- und das bis dato nicht operationalisierte Schuldenstandskriterium. ${ }^{64}$ Die Steuerung der Eurozone sollte nach französischer Vorstellung im Wesentlichen im Rahmen zwischenstaatlicher Kooperation in Form monatlich stattfindender Euro-Gipfel unter Ausschluss der Mitgliedstaaten umgesetzt werden, die die gemeinsame Währung noch nicht eingeführt haben. Dies knüpft an die Idee eines ,gouvernement économique ' an. ${ }^{65}$ Aus dieser Perspektive betrachtet kam der Widerstand des Vereinigten Königreichs gegen die Veränderung des Primärrechts zumindest nicht ungelegen, da er die Gelegenheit zur Absicherung eines aus den Euro-Staaten bestehenden ,Kerneuropas` bot und eine weitgehende Einbindung der Gemeinschaftsinstitutionen de-legitimierte. Die französische Regierung sah die festgeschriebenen Fiskalregeln mit Skepsis, machte sich diese jedoch - aus taktischen Gründen? - schon in einem gemeinsamen Brief von Angela Merkel und Nicolas Sarkozy vor den Verhandlungen zu eigen. ${ }^{66}$ Jedoch stand sie oder ,Paris' einer Verbindung von Fiskalvertrag und Europäischem Stabilisierungsmechanismus kritisch gegenüber und argumentierte, die im ESM-Vertrag verankerte Konditionalität sei ausreichend. ${ }^{67}$ Inwiefern schlägt sich die zuvor konstatierte relative Schwäche Frankreichs in den Verhandlungen nieder und inwiefern führt sie zu der Akzeptanz einer deutschen Hegemonie? Während der Verhandlungen

59 Nikolaus Busse: Rat und Tat in endlicher Zeit, in: Frankfurter Allgemeine Zeitung, 31.1.2012.

60 Kaczmarek: Ergebnisse des deutschen EU-Gipfels, 2012.

61 Nicolas Sarkozy: Rede in Toulon, 1.12.2011.

62 Arnaud Leparmentier/Philippe Ricard: Angela Merkel et Nicolas Sarkozy à la recherche d'un compromis, in: Le Monde, 6.12.2012.

63 Miguel Mora: Merkel y Sarkozy apuestan por un nuevo Tratado aunque no incluya a todos, in: El País, 5.12.2011; Jean-Jacques Mével/Patrick Saint-Paul: La France et l'Allemagne envisagent un plan d'union budgétaire, in: Le Figaro, 29.11.2011.

64 Werner Mussler/Manfred Schäfers: Union streitet vor dem EU-Gipfel über den Inhalt des Fiskalpakts, in: Frankfurter Allgemeine Zeitung, 30.1.2012.

65 Philippe Ricard: Dernières tractations avant le sommet des vingt-sept, in: Le Monde, 29.1.2012.

66 Peter Ludlow: The European Council of 8/9 December 2011, 2012, S. 13-19.

67 Kaczmarek: Ergebnisse des deutschen EU-Gipfels, 2012. 
zeigte sich eine enge Zusammenarbeit der deutschen und französischen Regierung, die sich am sichtbarsten in einem gemeinsamen Brief an den Präsidenten des Europäischen Rates manifestiert. Die Analyse der Verhandlungspositionen weist auf signifikante Unterschiede hin; während Deutschland darauf drängte, nationale Souveränität einzuschränken, um fiskalische Defizite zu vermeiden, ist der Erhalt der Handlungsmacht nationaler Regierungen Kernelement der französischen Position. Es mag als Beleg der Effektivität des deutsch-französischen Tandems gesehen werden, dass es trotz der anfänglich stark divergierenden Standpunkte eine gemeinsame Position gefunden wurde, wie sie im bereits erwähnten Brief an Herman von Rompuy ausgedrückt wird.

\section{Spanien - auf der Suche nach Orientierung}

Welche Agenda verfolgte das von der Krise gebeutelte Spanien während der Verhandlungen zum Fiskalvertrag? Folgte es in der Tat Deutschland? Spaniens Wirken stand im Spannungsfeld zwischen dem Regierungswechsel und der Notwendigkeit, das Vertrauen der Märkte in spanische Anleihen wieder herzustellen. Der Forderung nach einer, goldenen Re-

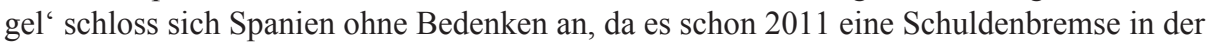
nationalen Verfassung etabliert hatte. Somit ergab sich auch kein Problem aus der Verknüpfung von Fiskalvertrag und Europäischem Stabilisierungsmechanismus. Vielmehr bot sich die Gelegenheit, Spanien als ,Musterschüler' darzustellen, der sich nach Kräften bemüht, den Haushalt zu konsolidieren. ${ }^{68}$ Die gleiche Argumentation fand Anwendung bei Spaniens Bereitschaft, einer strikten supranationalen Überwachung der Sparvorgaben zuzustimmen. ${ }^{69}$ Das Sanktionsverfahren der , umgekehrten` qualifizierten Mehrheit wurde zudem als besser handhabbar bewertet als der Entzug von Stimmrechten oder andere diskutierte Sanktionsmöglichkeiten. ${ }^{70}$ Einer Vertragsänderung stand die spanische Regierung kritisch gegenüber, da das Verfahren als zu langwierig und schwierig angesehen wurde. ${ }^{71}$ Ein Vertragsabschluss unter Nichtbeteiligung einiger Mitgliedstaaten wurde von der spanischen Regierung akzeptiert, solange er nicht die Gefahr eines Ausschlusses Spaniens enthielt. ${ }^{72}$ Einen größeren Konfliktpunkt mit Deutschland stellte das spanische Drängen nach Beseitigung makroökonomischer Ungleichgewichte dar. ${ }^{73}$ Diesem Narrativ folgend ist die ,unvollendete Wirtschafts- und Währungsunion einer der maßgeblichen Gründe für die spanischen Probleme. Folglich befürwortete Spanien mehr Kompetenzen für supranationale Institutionen nicht nur zur Überwachung nationaler Fiskalpolitik, sondern auch in der Wirtschaftspolitik. ${ }^{74}$ Das traditionell stark ausgeprägte normative Bekenntnis Spaniens zur Europäischen Union mit supranationaler Ausrichtung bildet einen Erklärungsansatz für den Willen Spaniens, sich verstärkter Überwachung zu fügen. Hinzu kommt die ebenfalls oben erläuterte dramatische wirtschaftliche Lage, die es zur Notwendigkeit macht, mit dem Fiskalvertrag

68 Mariano Rajoy: Conferencia de prensa de la canciller de la República Federal de Alemania y del presidente del Gobierno, 26.1.2012, abrufbar unter: http://www.lamoncloa.gob.es/Presidente/Intervenciones/Conferenciasde Prensa/2012/prrp20120126.htm (letzter Zugriff: 17.12.2012).

69 Rajoy: Discurso de Mariano Rajoy en la sesión de investidura como presidente del Gobierno, 19.12.2011, abrufbar unter: http://www.lamoncloa.gob.es/Presidente/Intervenciones/Sesionesparlamento/2011/191211Dis cursoInvestidura.htm (letzter Zugriff: 28.1.2013).

70 Dinan: Government and Institutions, 2011, S. 109.

71 Carlos E. Cué/Miguel González: España quiere tener poder de veto en el futuro fondo de rescate europeo, in: El País, 8.12.2011.

72 Andreu Missé/Claudi Pérez: La noche en que Londres unió a Europa, in: El País, 11.12.2012, S. 3; Mestres: The View from Spain, 2012, S. 18.

73 Agence Europe: Budget Talks Make Good Progress, 11.1.2012.

74 Süddeutsche Zeitung: Zapatero wünscht sich „deutscheres“Europa, Interview mit José Luis Zapatero, 2.2.2011. 
ein Signal an die Märkte zu senden. Deutschland ist das Rollenmodell der spanischen Reformen, weshalb schon die anfänglichen Verhandlungspositionen große Übereinstimmung aufweisen und die Akzeptanz deutscher Hegemonie andeuten.

\section{Irland - nicht noch ein Referendum!}

Welche Prioritäten setzte die irische Regierung? Waren diese kompatibel mit der deutschen Agenda? Wenngleich die irische Regierung der Notwendigkeit nach Haushaltskonsolidierung, wie im Fiskalvertrag gefordert, nicht widersprach, versuchte sie unter allen Umständen ein Referendum über den Vertrag zu vermeiden. Dieses Anliegen war unvereinbar mit dem deutschen Drängen nach einer Änderung des Primärrechts; zudem wandte sich die irische Regierung gegen die Festschreibung der ,goldenen Regel' in nationalen Verfassungsordnungen. ${ }^{75}$ Sie bevorzugte eine Lösung im Kreise der ,EU-27 ${ }^{\text {}}$, da diese eine Einbindung des Vereinigten Königreichs bedeutet hätte, das laut Kenny der „engste Verbündete“ ${ }^{76}$ Irlands ist. Jedoch betraf der Widerstand lediglich die Form, nicht den Inhalt. In Irland herrschte unter den politischen Eliten ein Konsens über die Notwendigkeit der fiskalischen Konsolidierung. ${ }^{77}$ Greift man auf die obigen Ausführungen zur Selbstwahrnehmung Irlands zurück, kann dies als Ausdruck der irischen Akzeptanz weitreichender Reformschritte verstanden werden. Ähnlich wie im Falle Spaniens war auch Irland zu einer strikten Überwachung der vereinbarten Sparziele durch die Kommission und den Gerichtshof willens, um das Vertrauen der Märkte zurückzugewinnen. ${ }^{78}$ Eine dauerhafte, auch nach Lösung der Schuldenkrise weiterbestehende Kompetenz der Haushaltskontrolle für Gemeinschaftsinstitutionen sollte allerdings vermieden werden. Irland strebte eine Begrenzung des Souveränitätstransfers auf möglichst wenige Kompetenzen an, ohne die Notwendigkeit eines gewissen Kompetenztransfers auf die europäische Ebene abzustreiten. Der Bezug zur Notwendigkeit von Maßnahmen zur Stimulierung des wirtschaftlichen Wachstums fand die Unterstützung Irlands, da die irische Regierung stets dafür plädierte, Haushaltsregeln mit einem Wachstumsprogramm zu kombinieren. ${ }^{79}$ Aufgrund der realen Gefahr, ein weiteres Hilfspaket beantragen zu müssen, und dem ungewissen Ausgang eines eventuellen Referendums über den Fiskalvertrag, wandte sich Irland gegen die Verknüpfung von Fiskalvertrag und ESM. ${ }^{80}$ Die divergierenden Positionen zu Deutschland weisen darauf hin, dass Irland einer Hegemonie Deutschlands mit Vorbehalten begegnet, ohne sich jedoch dem mächtigen Ein-

75 Kaczmarek: Ergebnisse des deutschen EU-Gipfels, 2012.

76 Enda Kenny: Statement by the Taoiseach on the European Council, Brussels 8/9 December. Dáil Éireann, 14 December 2011, abrufbar unter: http://www.merrionstreet.ie/index.php/2011/12/statement-by-the-taoiseach-o n-the-european-council-brussels-89-decemberdail-eireann-14-december-2011/?cat=70 (letzter Zugriff: 24.1.2013).

77 Peadar Kirby: Wahlanalyse Irland 2011. Im Angesicht wütender Wähler: Die verzweifelten Überzeugungsversuche irischer Politiker, Friedrich-Ebert-Stiftung: Internationale Politikanalyse, Berlin 2011, S. 7.

78 Eamon Gilmore: Statement by Tánaiste Mr. Eamon Gilmore T.D. on the Thirtieth Amendment of the Constitution (Treaty on Stability, Coordination and Governance in the Economic and Monetary Union) Bill 2012, 18.4.2012, abrufbar unter: http://www.merrionstreet.ie/index.php/2012/04/statement-by-tanaiste-mr-eamongilmore-t-d-on-the-thirtieth-amendment-of-the-constitution-treaty-on-stability-coordination-and-governancein-the-economic-and-monetary-union-bill-2012/?cat=70 (letzter Zugriff: 20.1.2013); Arthur Beesley: Fate of the Euro, in: The Irish Times, 3.12.2011.

79 Enda Kenny: Statement by the Taoiseach on the meeting of the European Council, Brussels, 28/29 June, 26.6.2012, abrufbar unter: http://www.merrionstreet.ie/index.php/2012/06/statement-by-the-taoiseach-on-themeeting-of-the-european-council-brussels-2829-june/?cat=70 (letzter Zugriff: 26.1.2013).

80 Kathy Sheridan: Yeah, but no, but yeah, but no, in: Irish Times, 28.4.2012. 
fluss Deutschlands gänzlich entziehen zu können, und einige Standpunkte denen Deutschlands letztlich anglich.

\section{Auswertung: Der Fiskalvertrag - ein Ausdruck deutscher Hegemonie?}

Welche Regierung setzte ihre Präferenzen durch? Diese Frage wird durch einen Vergleich der zuvor geschilderten Verhandlungspositionen mit dem Fiskalvertrag in seiner beschlossenen Fassung beantwortet. Es wird davon ausgegangen, dass eine hohe Übereinstimmung zwischen der deutschen Verhandlungsagenda und dem Fiskalvertrag als Beleg einer Hegemonie gewertet werden kann. Ein Vergleich der Standpunkte anderer Mitgliedstaaten mit den deutschen erlaubt zudem Rückschlüsse auf die Wahrnehmung und Überzeugungsfähigkeit Deutschlands gegenüber anderen Mitgliedstaaten. Die Ergebnisse können aufgrund der geringen Anzahl der ausgewählten Staaten, der Fokussierung auf nur ein Politikfeld und in Ermangelung weiterer Fallbeispiele nur unter einem gewissen Vorbehalt verallgemeinert werden.

Es werden neun Kriterien aufgestellt, die entweder aus dem Vertragstext hervorgehen oder maßgebliche Inhalte des Verhandlungsprozesses darstellen. Erstens zeigt sich, dass Deutschland treibender Faktor hinsichtlich einer Vertragsänderung war: „Ohne [Merkels] Druck hätte niemand eine Vertragsänderung auch nur in Erwägung gezogen“. ${ }^{81}$ Zwar kam es durch das Veto des Vereinigten Königreichs zu keiner Vertragsänderung, allerdings zieht auch ein intergouvernementaler Vertrag den gewünschten Effekt des ,Zeichensetzens' nach sich. Frankreich schwenkte schon früh auf die deutsche Linie ein, Spanien stand der Frage eher indifferent gegenüber, Irland allerdings begegnete dem Vorschlag aufgrund der Furcht vor einem erneuten Referendum mit großen Vorbehalten. ${ }^{82}$ Die Durchsetzung eines Vertrags außerhalb des rechtlichen Fundaments der Europäischen Union ohne Zustimmung durch das Vereinigte Königreich zeigt die Durchsetzungsfähigkeit der Bundesregierung bei den Verhandlungen.

Zweitens wird die reduzierte Anzahl der teilnehmenden Staaten betrachtet. Zwar hätte die Bundesregierung ein Abkommen im Kreise aller 27 Mitgliedstaaten bevorzugt, ein ,EU-17+'-Vertragsabschluss stellte aber immerhin die zweitbeste Lösung dar. Frankreich sah sich mit dieser Lösung der Vision eines Kerneuropas einen Schritt näher, während Irland insbesondere die Nicht-Teilnahme des Vereinigten Königreichs bedauerte. Spanien unterstützte eine Einigung auch ohne Einwilligung aller Mitglieder.

Als dritte Kategorie wird die Verpflichtung der Unterzeichnerstaaten auf eine ausgeglichene Haushaltsregel herangezogen. Diese Forderung stellt den Kern der deutschen Verhandlungsposition dar. Der Schlüssel zur Durchsetzung war eine frühe Einigung mit Frankreich, das die Forderung - auch gegen den erbitterten Widerstand Irlands - unterstützte. Ein Zugeständnis war allerdings die Formulierung, dass die Verankerung der ,Schuldenbremse nicht zwingend, ${ }^{83}$ sondern nur ,Vorzugsweise ${ }^{\text {c84 }}$ in der Verfassung oder auf verfassungsähnlicher Ebene stattzufinden habe. Dennoch kann die weitgehende Durchsetzung der deutschen Forderung als weiterer Beleg für die regelsetzende Autorität Deutschlands gewertet werden.

81 Peter Ludlow: The European Council of 8/9 December 2011, 2012, S. 39.

82 Ebenda, S. 21.

83 Europäischer Rat: Erklärung der Staats- und Regierungschefs des Euro-Währungsgebiets, Brüssel, den 9. Dezember 2011.

84 Art. 3 Abs. 2 Fiskalvertrag. 
Viertens kristallisierte sich die Zuständigkeit supranationaler Institutionen als Streitpunkt heraus. Strittig war die Frage, wie weitgehend die Kompetenzen des Gerichtshofs in dem Vertrag definiert werden sollten. Laut der Endfassung des Vertragstextes kontrolliert der Gerichtshof, ob die Schuldenbremsen in nationales Recht überführt werden. Allerdings überprüft er nicht, ob das Defizit- und Schuldenkriterium eingehalten wird. Letzteres betreffend scheiterten Deutschland und Spanien insbesondere an französischem Widerstand. Allerdings wurde auf deutschen Druck hin vereinbart, dass die Trio-Präsidentschaft des Rates einen Antrag im Rat stellt, um ein Sanktionierungsverfahren in Gang zu setzen, sobald ein Mitgliedstaat die Kriterien verletzt. ${ }^{85}$ Auch Irland konnte einer stärkeren Überwachung durch den Gerichtshof zustimmen. Die letztlich gefundene Lösung stellte in erster Linie einen Kompromiss zwischen den Positionen Deutschlands und Frankreichs dar, woran einmal mehr deren Schlüsselrolle für die Verhandlungen sichtbar wird.

Fünftens entzündeten sich größere Debatten an den der Kommission übertragenen Kompetenzen. Während Deutschland das ursprüngliche Ziel, die Kommission mit umfassenden Klagerechten auszustatten, aufgrund rechtlicher Bedenken rasch aufgab, stand Frankreich zu jeglichen Vorstößen in diese Richtung in strikter Opposition. Spanien hingegen befürwortete derlei Maßnahmen im Rahmen einer verstärkt supranationalen institutionellen Architektur (siehe unten). Letztlich konnte Frankreich seine Präferenzen durchsetzen, sodass die Kommission laut Vertrag kein Klagerecht besitzt.

Sechstens stellte sich die Frage nach einem adäquaten Sanktionsverfahren. Deutschland befürwortete das Prinzip der ,umgekehrten' qualifizierten Mehrheit, während Frankreich Legimitationsprobleme sah und für die Beibehaltung der konventionellen qualifizierten Mehrheit plädierte. Spanien und Irland hingegen standen vereinfachten Defizitverfahren nicht im Weg, galt es doch, noch drastischere Sanktionen wie beispielsweise den Entzug von Stimmrechten zu verhindern. ${ }^{86}$ In diesem Punkt setzte sich Deutschland mit seiner Position durch. Beachtenswert ist die ohnehin nicht sehr weitgehende Forderung Deutschlands nach ,umgekehrter' qualifizierter Mehrheit, die ja keinen Automatismus vorschreibt. Auch hier zeigt sich die Bedeutung des deutsch-französischen Tandems, die Verhandlungen zu prägen.

Siebtens entsprechen die Vereinbarungen zur institutionellen Architektur weitgehend französischen Präferenzen. Sie kann als Wiederaufgreifen der ,französischen“ Idee eines ,gouvernement économique interpretiert werden. Deutschland hingegen drängte erfolgreich auf die partielle Integration der Staaten, die nicht die gemeinsame Währung eingeführt haben, in die Euro-Gipfel. Von Spanien kamen hingegen Impulse für eine stärker supranationale Integration des Währungsgebiets, während die irische Regierung zwischen der Notwendigkeit einer stärkeren Überwachung zur Beruhigung der Märkte und dem Reflex nationaler Souveränität keine starke Position entwickeln konnte. Hier zeigt sich sehr deutlich die bereits zuvor erläuterte Präferenz Frankreichs für ein nach intergouvernementalen Prinzipien vollzogenes Integrationsmodell. Zieht man die Relevanz dieses Aspekts der Vereinbarung für die zukünftige Steuerung der Eurozone in Betracht, so sind diese Bestimmungen zur institutionellen Architektur offensichtlich als ein elementarer Verhandlungserfolg der französischen Regierung zu werten.

85 Anna-Lena Kirch/Daniela Schwarzer: Die Ratifizierung des Fiskalpakts und des ESM in den Ländern der Eurozone - Rechtliche und politische Rahmenbedingungen, Stiftung Wissenschaft und Politik: SWP-Arbeitspapier, 2012, S. 6.

86 Peter Ludlow: The European Council of 8/9 December 2011, 2012, S. 33-35; Dinan: Government and Institutions, 2011, S. 109. 
Achtens war die Aufnahme einer Formulierung, die die Notwendigkeit von Wachstum betonte und Wege definierte, dieses zu erzielen, unter den Verhandlungsparteien außerordentlich strittig. Deutschland setzte sich durch, jegliche Hinweise auf ein nachfrageorientiertes, kostspieliges Wachstumsprogramm zu vermeiden. Über die unverbindliche Formulierung, zu Wachstum „bei[zu]tragen“" 87 hinaus gehen die Mitgliedstaaten keine weiteren Verpflichtungen ein. Spanien und Irland hätten eine konkrete „Wachstumsagenda“ bevorzugt, doch eine Wachstumsinitiative zu spezifizieren erwies sich als zu kontrovers, um eine Einigung zu erzielen. ${ }^{88}$

Neuntens war die Verknüpfung von Europäischem Stabilisierungsmechanismus und Fiskalvertrag bis zuletzt vieldiskutierter Verhandlungspunkt. Deutschland setzte seine Position durch, sodass laut Erwägungsgründen des Fiskalvertrags dessen Ratifizierung Bedingung für den Erhalt von ESM-Mitteln ist. Jeglicher Widerstand - maßgeblich auch von Frankreich - konnte Deutschland nicht zum Verzicht hierauf bewegen. ${ }^{89}$ Beide Vertragswerke zu verbinden war eine deutsche Kernposition, um die Konditionalität eventueller Hilfszahlungen noch einmal zu unterstreichen. Irland konnte die Verknüpfung vor dem Hintergrund der eigenen Fiskalsituation nur schwer akzeptieren; Spanien dagegen hatte bereits zuvor eine nationale Schuldenbremse etabliert und konnte die Forderung somit unterstützen.

Die folgende Tabelle fasst die Verhandlungspositionen zusammen. Inwiefern das Verhandlungsergebnis der deutschen Position entspricht, wird in der Spalte ,Deutschland ' ausgedrückt. Inwiefern die Positionen der drei anderen Mitgliedstaaten mit den deutschen Präferenzen übereinstimmen, wird aus einem Vergleich der Spalte ,Deutschland' mit den Spalten ,Frankreich', ,Spanien“ und ,Irland' ersichtlich. ,++' und ,--' stellen die Extrempunkte einer Skala dar: der Erstere drückt aus, dass sich die Präferenzen eines Mitgliedstaates (nahezu) unverändert in der entsprechenden Vertragsbestimmung niederschlagen; letzterer zeigt an, dass ein Mitgliedstaat mit seiner Position gescheitert ist. ,0` bedeutet, dass der Staat sich weitgehend indifferent zeigte.

Vieles spricht demnach für die Betrachtung des Fiskalvertrags als Produkt deutscher Präferenzen. Ersichtlich wird jedoch auch die erfolgreiche Verhandlungsführung Frankreichs. Diese beiden Tatsachen weisen den Weg zu einer Interpretation des Fiskalvertrags als Resultat deutsch-französischer Kooperation beziehungsweise des Eingehens auf die Interessen des anderen. In drei von neun Fällen entsprach das Verhandlungsergebnis deutschen Forderungen $\left(,++^{\circ}\right)$. In fünf Fällen spiegelt es weitgehend die deutsche Position wider $\left(,+^{\circ}\right)$. Nur bei einem Vertragselement musste Deutschland eine seiner Verhandlungsagenda widersprechende Formulierung in Kauf nehmen $\left(,-^{-}\right)$. In vier Fällen reflektiert der Fiskalvertrag französische Interessen nahezu ohne Einschränkung $\left(,++^{\circ}\right)$, zwei weitere Elemente befinden sich im breiteren Interessenfeld Frankreichs $\left(,+^{\circ}\right)$. Allerdings widerspricht der Fiskalvertrag in drei Fällen und damit signifikant häufiger als im Falle Deutschlands den nationalen französischen Positionen (,-' oder ,-- - ). In sieben Fällen stimmen die Vertragselemente mit Spaniens Position vollständig oder teilweise $\left(,++^{\text {' }}\right.$ oder,$\left.+^{\circ}\right)$ überein, in zwei Fällen widerspricht er spanischen Standpunkten (,-'). Irland musste die meisten Konzessionen leisten: zwar befinden sich drei der untersuchten Elemente im Bereich irischer Standpunkte, jedoch war es viermal mit dem Verhandlungsergebnis nicht zufrieden (,-' oder ,--').

87 Art. 1 Abs. 1 Fiskalvertrag.

88 Peter Ludlow: The European Council of 8/9 December 2011, 2012, S. 13.

89 Werner Mussler: Nicht alle Streitpunkte zum EU-Fiskalpakt sind geklärt, in: Frankfurter Allgemeine Zeitung, 12.1.2012. 
Tabelle 2: Übersicht der Präferenzen zu den Verhandlungen zum Fiskalvertrag

\begin{tabular}{|l|c|c|c|c|}
\hline \multirow{2}{*}{$\begin{array}{l}\text { Vertragsbestimmung/ } \\
\text { Verhandlungselement }\end{array}$} & \multicolumn{3}{|c|}{$\begin{array}{r}\text { Ausmaß zu dem Fiskalvertrag die Präferenzen von ... } \\
\text { reflektiert }\end{array}$} \\
\cline { 2 - 5 } & Deutschland & Frankreich & Spanien & Irland \\
\hline Rechtsform der Maßnahmen & ++ & ++ & + & -- \\
\hline Anzahl der Mitgliedstaaten & + & ++ & + & - \\
\hline $\begin{array}{l}\text { Nationale Implementierung der } \\
\text {,goldenen Regel }\end{array}$ & + & + & + & - \\
\hline $\begin{array}{l}\text { Verbindung von ESM-Mitteln und } \\
\text { Fiskalvertrag-Ratifizierung }\end{array}$ & ++ & - & + & - \\
\hline Klagerecht für Kommission & + & ++ & - & 0 \\
\hline $\begin{array}{l}\text { Sanktionsbeschluss mit ,umgekehrter } \\
\text { QM/konventioneller QM }\end{array}$ & + & - & + & + \\
\hline Zuständigkeit des Gerichtshofs der EU & + & +- & + & + \\
\hline Institutionelle Architektur & + & ++ & + & + \\
\hline $\begin{array}{l}\text { Ausschluss nachfrageorientierter } \\
\text { Wachstumsprogramme }\end{array}$ & ++ & + & ++ & + \\
\hline
\end{tabular}

Quelle: Eigene Darstellung.

Schwierig ist die Frage zu beantworten, ob Deutschland die anderen Mitgliedstaaten auch tatsächlich überzeugt hat oder das Resultat nicht doch ,nur' das Ergebnis von Machtasymmetrien ist. Im Falle Frankreichs zeigt sich ein gemischtes Bild: Fünf gleichen bis ähnlichen Positionen stehen divergierende Standpunkte in vier Fällen gegenüber. Mit Spanien hingegen bestand weitgehend Interessenidentität: Mit einer Ausnahme vertraten beide Staaten identische bis ähnliche Positionen. Zwischen Irland und Deutschland taten sich die größten Unterschiede auf: In vier Fällen kam es zu Interessendivergenzen.

Es wird offensichtlich, dass das deutsch-französische Tandem aller Unkenrufe zum Trotz noch erhebliche Durchsetzungsfähigkeit besitzt: Wenn Deutschland und Frankreich die gleiche Position vertraten und diese mit einer hohen Priorität versahen (beide,$++^{\circ}$ ), wurde dieser Standpunkt letztlich auch angenommen (siehe Zeile ,Rechtsform der Maßnahmen'). Zweitens ist weitgehende Interessengleichheit (einmal,$++^{\text {' }}$ und einmal,$+^{\circ}$ ) ausreichend, um deutsch-französische Vorschläge auch gegen Widerstand durchzusetzen (siehe Zeilen ,Anzahl der Mitgliedstaaten“, ,Nationale Implementierung der ,goldenen Regel““, ,Institutionelle Architektur', ,Ausschluss nachfrageorientierter Wachstumsprogramme“). Drittens wird erkennbar, dass bei Interessendivergenz zwischen Deutschland und Frankreich (einmal ,+' oder ,++', einmal ,--' oder ,--') während den Verhandlungen Deutschland durchsetzungsfähiger agierte (siehe Zeilen, Verbindung von ESM-Mitteln und Fiskalvertrag-Ratifizierung', ,Sanktionsbeschluss mit ,umgekehrter“ QM/konventioneller QM“, ,Zuständigkeit des Gerichtshofs der EU'). Dies lässt sich als eine Differenzierung innerhalb des deutschfranzösischen Tandems interpretieren: Deutschland vertritt die eigenen Interessen erfolgreicher als Frankreich. Wenngleich beide Staaten maßgeblich die Inhalte des Vertrags mitbestimmten, bildet das Verhandlungsergebnis offenbar die Asymmetrie innerhalb des Duos

90 Qualifizierte Mehrheit. 
ab. Dieses Ergebnis der Fallstudie stützt die These einer deutschen Hegemonie allerdings nur bedingt. Eine Charakterisierung Frankreichs als ein Deutschland folgender Verhandlungsteilnehmer wird der französischen Rolle nicht gerecht. Naheliegender wäre es, das Ergebnis als Beleg für eine deutsch-französische Hegemonie zu werten. Beginnend mit dem erwähnten gemeinsamen Brief von Merkel und Sarkozy an van Rompuy setzten Deutschland und Frankreich die Agenda und wiesen mit einer Kompromisslösung bei strittigen Punkten den Weg zu einer Einigung im Kreise der anderen Mitgliedstaaten. Indem Deutschland die Regeln zur Fiskalpolitik durchsetzte und Frankreich die institutionelle Architektur der Eurozone nach eigenen Vorstellungen prägen konnte, erwies sich das deutsch-französische Tandem als führend bei den Verhandlungen.

\section{Eine Hegemonie mit Einschränkungen}

Ist Deutschland ein Hegemon in der Europäischen Union? Die Analyse der drei Dimensionen von Hegemonie nach Triepel - materielle Fähigkeit zu Hegemonie, Wille des Hegemons zur Wahrnehmung einer hegemonialen Rolle sowie die Akzeptanz des Hegemons unter den ihm folgenden Staaten - am Beispiel des Fiskalvertrags erbringt unterschiedliche Ergebnisse.

Erstens wird deutlich, dass Deutschland aus der Position eines systemrelevanten Akteurs in der Europäischen Union agiert. Dieser Status basiert sowohl auf der Größe der Volkswirtschaft als auch auf der relativ guten Wirtschaftslage während der Schuldenkrise, die sich nicht zuletzt in einem durchgängigen ,AAA'-Rating widerspiegelt. Letzteres Kriterium hebt Deutschland dann auch deutlich von Frankreich ab. Die materielle Dimension legt daher theoretisch und faktisch den Grundstein einer deutschen Hegemonie in der Europäischen Union.

Zweitens bestehen jedoch Zweifel, ob Deutschlands hegemoniale Stellung in ausreichendem Maße Eingang in die Identität des Staates gefunden hat, um eine hegemoniale Rolle auszufüllen. Es scheint, als laste noch immer der Schatten der Vergangenheit auf der deutschen Rolle; folglich mag es nicht überraschen, dass aus Kreisen der Bundesregierung eine unilateral wahrgenommene Hegemonie mit dem Hinweis auf die Geschichte abgelehnt wird. Zwar mag die Initiative zum Fiskalvertrag als Beleg für ein Bewusstsein für die eigene Macht gewertet werden. Dies steht jedoch im Kontext mit der Fokussierung auf Frankreich, dessen hoher Stellenwert in den Verhandlungen schon zu deren Beginn durch den gemeinsamen Brief von Merkel und Sarkozy signalisiert wird.

Drittens bestehen Zweifel an der Akzeptanz einer hegemonialen Rolle Deutschlands durch die anderen Mitgliedstaaten. Die Ergebnisse zu Frankreich, Spanien und Irland variieren beträchtlich. Die Analyse verdeutlicht, dass eine deutsche Hegemonie mit Frankreichs Selbstwahrnehmung nur schwer vereinbar ist. Auch im Fiskalvertrag konnte Frankreich wichtige Präferenzen - teils auch gegen den Willen Deutschlands - durchsetzen. Im Falle Spaniens hingegen wurde eine weitgehende Akzeptanz deutscher Hegemonie deutlich. Die neugewählte Regierung war in ihrer Anfangsphase sehr stark an Deutschland orientiert. Es stellte zeitweise sogar das Rollenmodell Spaniens dar und die Regierung übernahm viele der deutschen Verhandlungspositionen. Die nunmehr unüberhörbare Kritik an deutschen Positionen fand sich im Untersuchungszeitraum noch nicht im spanischen Diskurs wieder. Im Falle Irlands lassen sich verschiedene Reibungspunkte zur deutschen Position erkennen. Ursächlich hierfür war das nachdrückliche Bestreben Irlands, ein Referendum zu vermeiden. 
Zusammenfassend belegt die vorliegende Studie, dass nur mit Einschränkungen von einer deutschen Hegemonie in der Europäischen Union gesprochen werden kann. Eine Interpretation des Fallbeispiels legt vielmehr eine deutsch-französische Hegemonie nahe. Zwar unternahm die Bundesregierung die Initiative zum Fiskalvertrag, jedoch wäre ohne die Unterstützung Frankreichs die (faktische) Nichtberücksichtigung des Vetos des Vereinigten Königreichs gegen eine Vertragsänderung schwerlich denkbar. Während der Verhandlungen waren die Positionen Deutschlands und Frankreichs in ähnlichem Maße entscheidend. Allerdings zeigte sich eine Differenzierung innerhalb des Tandems, die - zumindest im untersuchten Politikfeld der Wirtschafts- und Währungspolitik - Deutschland eine einflussreichere Rolle zuweist. Es bleibt abzuwarten, ob sich im weiteren Verlauf der Krise die Kräfteverhältnisse weiter zugunsten Deutschlands verschieben; mit dem Reformstau in Frankreich, der latent unsicheren politischen Situation in Italien und Spanien sowie der manifesten Skepsis gegenüber dem europäischen Einigungsprojekt im Vereinigten Königreich bestehen auch zukünftig zahlreiche Faktoren, die vielfältige Wirkungen auf die deutsche Rolle in der Europäischen Union entwickeln könnten.

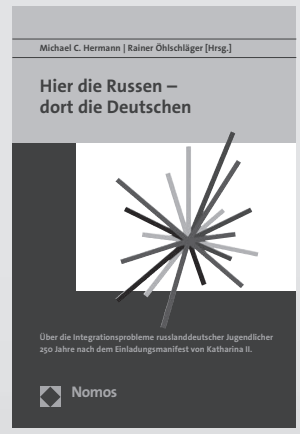

\section{Hier die Russen - dort die Deutschen}

Über die Integrationsprobleme russlanddeutscher Jugendlicher 250 Jahre nach dem Einladungsmanifest von Katharina II.

Herausgegeben von Michael C. Hermann und Rainer Öhlschläger

2013, 120 S., brosch., 22,- $€$

ISBN 978-3-8487-0511-5

Die Integration von Russlanddeutschen verlief schwieriger als angenommen und ist bis heute ein Thema. Die Autoren dieses Bandes fragen nach der Situation russlanddeutscher Jugendlicher in Deutschland und Russland und suchen dabei auch den interkulturellen Dialog mit Wissenschaftlern aus der Russischen Föderation.

Bestellen Sie jetzt telefonisch unter 07221/2104-37. Portofreie Buch-Bestellungen unter www.nomos-shop.de/20996

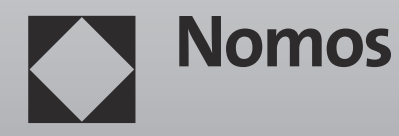

